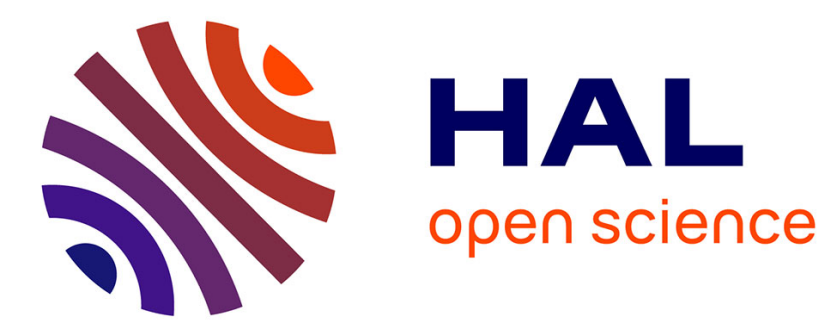

\title{
Set theory based condition in LMI form for network topology preservation for decentralized control
}

Mirko Fiacchini, Irinel-Constantin Morarescu

\section{To cite this version:}

Mirko Fiacchini, Irinel-Constantin Morarescu. Set theory based condition in LMI form for network topology preservation for decentralized control. 51st IEEE Conference on Decision and Control, CDC 2012, Dec 2012, Maui, Hawaii, United States. pp.CDROM. hal-00733793

\section{HAL Id: hal-00733793 \\ https://hal.science/hal-00733793}

Submitted on 19 Sep 2012

HAL is a multi-disciplinary open access archive for the deposit and dissemination of scientific research documents, whether they are published or not. The documents may come from teaching and research institutions in France or abroad, or from public or private research centers.
L'archive ouverte pluridisciplinaire HAL, est destinée au dépôt et à la diffusion de documents scientifiques de niveau recherche, publiés ou non, émanant des établissements d'enseignement et de recherche français ou étrangers, des laboratoires publics ou privés. 


\title{
Set theory based condition in LMI form for network topology preservation for decentralized control
}

\author{
Mirko Fiacchini and Irinel-Constantin Morărescu *
}

September 19, 2012

\begin{abstract}
Connectivity preservation of the interconnection network is an essential ingredient in decentralized control. This paper focuses on the preservation of a given network topology which ensures the connectivity. We consider a networked system with a discrete dynamics in which two subsystems are able to communicate if an algebraic relation between their states is satisfied. The connected subsystems are called neighbours. The subsystems updates their state in a decentralized manner by taking into account their neighbours state. Each connection is preserved as far as the algebraic relation is verified. The objective of the proposed set theory based control law is to keep the state of each subsystem in a specific domain that ensures the algebraic constraint satisfaction. The resulting control design reduces to the solution of convex problems involving Linear Matrix Inequalities (LMI), then easily solvable. Beside the network preservation, the global coordination may be imposed by adding supplementary constraints in the control design.
\end{abstract}

\section{Introduction}

The control of the large scale systems in centralized framework needs huge computation and communication capabilities which are very expensive. The alternative is to implement control laws in a decentralized manner which is scientifically challenging but less costly. The main applications concern the decentralized communication $[1,15,17]$, decentralized control [21,30,31], distributed optimization [28,35,36] and analysis of social networks $[12,13,20,27]$.

A closely related area is that of consensus, flocking and multi-agent systems. Most of the works on consensus and coordination are based on bi-directional information exchange between neighbouring nodes (leading to undirected communication graphs)

\footnotetext{
* Mirko Fiacchini and Irinel-Constantin Morărescu are with the Université de Lorraine, CRAN, UMR 7039 and the CNRS, CRAN, UMR 7039, 2 av. de la forêt de Haye, Vandoeuvre-lès-Nancy, 54500, France, mirko.fiacchini@univ-lorraine.fr, constantin.morarescueuniv-lorraine.fr
} 
with rigorous convergence proofs given in [21]; this has been extended to include directed communication graphs, for instance in [26,30,31]. Further generalizations of the problem allowed for the inclusion of agent dynamics (typically linear, second order systems) in the consensus problem, [29], which play an important role in networks of mobile agents that move with finite dynamics.

It is noteworthy that the topology of the communication network between the interconnected subsystems is always essential in the decentralized framework. In [24] the authors derived sufficient conditions on the initial state of interconnected double integrators that guarantee the connectivity of the interconnection network and thus assure the flocking.

In this paper we present sufficient conditions for network topology preservation based on set theory and convex analysis. In the context of control and dynamical systems analysis, set theory has been employed since the end of the sixties. The seminal work [6] focusses on the characterization of invariance for discrete time systems. Some notable contributions on invariant set analysis and computation have appeared in recent years, see $[9,10,22]$, and the monograph [11] on set theory in control and invariance. The characterization of invariant subspaces, strongly related also with the properties of controllability and observability, has been treated in [2-4]. The set membership estimation approach for systems with unknown but bounded uncertainty should be mentioned, see the pioneering works $[8,34,38]$ and the more recent $[18,23]$. In the context of robust control and constrained control, the basis of the worst case approach have been posed in $[7,19,37]$ and give raise to modern prediction based control techniques, see the survey on Model Predictive Control [25]. One of the main benefits of set theoretic methods, is the fact that they permits to apply results proper of convex analysis, which often lead to computational affordable solutions of the considered problems. The notable contributions on convex analysis and convex optimization provided in $[5,14,16,32,33]$ are usually a solid basis for the application of set theory in control.

We consider a networked system with a discrete dynamics in which two subsystems are able to communicate if an algebraic relation between their states is satisfied. The connected subsystems are called neighbours. The subsystems updates their state in a decentralized manner by taking into account their neighbours state. Each connection is preserved as far as the algebraic relation is verified. Thus, we design a decentralized control law that ensures the satisfaction of the algebraic constraint. As will be shown, the set theory based condition ensuring the topology preservation reduces to solving a convex optimization problem in LMI form. Therefore, we not only propose a new tool for decentralized control but also an easy implementable one.

The paper is organized as follows. In Section II the problem under analysis is presented. The main results concerning the conditions for network topology preservation are provided in Section III and applied to an illustrative example in Section IV. The paper terminates with concluding remarks and further works ideas.

\section{Notation}

The set of positive integers smaller than or equal to the integer $n \in \mathbb{N}$ is denoted as $\mathbb{N}_{n}$, i.e. $\mathbb{N}_{n}=\{x \in \mathbb{N}: 1 \leq x \leq n\}$. Given a symmetric matrix $P \in \mathbb{R}^{n \times n}$, notation $P>0$ 
$(P \geq 0)$ means that $P$ is positive (semi-)definite, as usual.

\section{Problem statement}

Consider a distributed system composed by $N \geq 2$ identical interconnected subsystems whose dynamics are given by

$$
x_{i}^{+}=A x_{i}+B u_{i}, \quad \forall i \in \mathbb{N}_{N},
$$

with $A \in \mathbb{R}^{n \times n}, B \in \mathbb{R}^{n \times m}$ and where $x_{i} \in \mathbb{R}^{n}$ is the current state, $x_{i}^{+}$the successor one and $u_{i} \in \mathbb{R}^{m}$ the control input of the $i$-th subsystem. In the sequel each subsystem is referred to as agent. Given a certain subset of connections $\mathscr{N}$, the objective is to design a decentralized control law ensuring that none of these connections are lost. In other words, the aim is to design the control actions $u_{i}(t)$ independently from $u_{j}(t)$, for all $i, j \in \mathbb{N}_{N}$ and $t \in \mathbb{N}$, such that every connection in $\mathscr{N}$ is maintained.

Let us suppose that the initial interconnection topology is given by the graph $G=$ $(\mathscr{V}, \mathscr{N}(0))$ where the vertex set is $\mathscr{V}=\mathbb{N}_{N}$ and the edge set $\mathscr{N}(0)$ connects the agents respecting a distance-like condition. Precisely, given the real scalar $r>0, d \in \mathbb{N}$ with $d \leq n$ and $T \in \mathbb{R}^{d \times n}$ such that $T T^{\top}$ invertible, the edge set at time $t$ is given by

$$
\mathscr{N}(0)=\left\{(i, j) \in \mathbb{N}_{N} \times \mathbb{N}_{N} \mid\left\|T\left(x_{i}(0)-x_{j}(0)\right)\right\|<r\right\} .
$$

Since $T$ is a matrix, i.e. a linear application, the graph $G$ is undirected (i.e. $(i, j) \in$ $\mathscr{N}(0) \Leftrightarrow(j, i) \in \mathscr{N}(0))$.

In the sequel we denote by $\mathscr{N} \subseteq \mathscr{N}(0)$ the set of undirected edges that must be preserved. For instance we derive the minimal robust graph as in [24] and we design the control that preserves all the edges of this graph. Therefore, we suppose that every agent $i$ knows the state of the $j$-th one if and only if $(i, j) \in \mathscr{N}$.

Definition 1 For all $i \in \mathscr{V}$ we define the set of neighbours of the $i$-th agent as

$$
\mathscr{N}_{i}=\left\{j \in \mathbb{N}_{N}:(i, j) \in \mathscr{N}\right\} .
$$

Thus, the objective can be formulated in terms of the error dynamics between neighbours, which is given by

$$
e_{i, j}^{+}=x_{i}^{+}-x_{j}^{+}=A\left(x_{i}-x_{j}\right)+B\left(u_{i}-u_{j}\right),
$$

for all $(i, j) \in \mathscr{N}$. In what follows the error dynamics between the $i$-th and the $j$-th agents will be referred to as $i j$ system. It is not difficult to see that, in the centralized case corresponding to $\mathscr{N}=\mathbb{N}_{N} \times \mathbb{N}_{N}$ the dynamics of the error can be imposed by an adequate choice of $u_{i}$ with $i \in \mathbb{N}_{N}$. For instance, taking $M \in \mathbb{R}^{m \times n}$ such that $\| A+$ $B M \|<1$ and defining the inputs by

$$
u_{i}=-M \sum_{j \in \mathbb{N}_{N}}^{j \neq i} x_{j}, \quad \forall i \in \mathbb{N}_{N}
$$


we obtain the $i j$ system in the form:

$$
e_{i, j}^{+}=(A+B M) e_{i, j} .
$$

Therefore, the error can be stirred to zero. Notice that $M$ exists if the dynamics associated to an agent is controllable.

However, in the decentralized framework the agent $i$ does not know the states of all the agents in the network but the effects of each input $u_{i}$ propagates into the whole network. Therefore, the $i j$ system is perturbed by the dynamics of all the agents different of $i$ and $j$, making difficult the design of the decentralized controls $u_{i}$, for $i \in \mathbb{N}_{n}$. As usual in multi-agent systems we consider the $i$-th input as the sum of terms proportional to the distances with its neighbours, that is

$$
u_{i}=\sum_{j \in \mathscr{N}_{i}} B K_{i, j}\left(x_{i}-x_{j}\right)=\sum_{j \in \mathscr{N}_{i}} B K_{i, j} e_{i, j},
$$

then we have that the $i j$ system results in

$$
e_{i, j}^{+}=A e_{i, j}+\left(B K_{i, j}+B K_{j, i}\right) e_{i, j}+\sum_{k \in \mathscr{N}_{i}}^{k \neq j} B K_{i, k} e_{i, k}-\sum_{k \in \mathscr{N}_{j}}^{k \neq i} B K_{j, k} e_{j, k},
$$

for all $i, j \in \mathbb{N}_{N}$. Hence, the dynamics of the system is given by matrix $A+B K_{i, j}+$ $B K_{j, i}$ if no perturbations due the presence of other agents would be present. Such perturbation is given by the terms in the summation, that is the perturbation on the $i j$ system is

$$
w_{i, j}=\sum_{k \in \mathscr{N}_{i}}^{k \neq j} B K_{i, k} e_{i, k}-\sum_{k \in \mathscr{N}_{j}}^{k \neq i} B K_{j, k} e_{j, k}
$$

and can be bounded within a set depending on the radius $r$ and on the knowledge of common neighbours of the $i$-th and $j$-th agents. Consider the sets

$$
\begin{aligned}
\mathscr{N}_{i, j} & =\mathscr{N}_{i} \cap \mathscr{N}_{j}, \\
\overline{\mathscr{N}}_{i, j} & =\mathscr{N}_{i} \backslash\left(\mathscr{N}_{i, j} \cup\{j\}\right), \\
\overline{\mathscr{N}}_{j, i} & =\mathscr{N}_{j} \backslash\left(\mathscr{N}_{i, j} \cup\{i\}\right),
\end{aligned}
$$

then, $\mathscr{N}_{i, j}$ denotes the common neighbours of the $i$-th and the $j$-th agents and $\overline{\mathscr{N}}_{i, j}$ the neighbours of the $i$-th one which are neither $j$ nor one of its neighbours, analogously for $\overline{\mathscr{N}}_{j, i}$. The elements of the set $\overline{\mathscr{N}}_{i, j} \cup \overline{\mathscr{N}}_{j, i}$ are referred to as the non-common neighbours of the $i j$ system. We define the cardinalities

$$
N_{i, j}=\left|\mathscr{N}_{i, j}\right|, \quad \tilde{N}_{i, j}=\left|\bar{N}_{i, j}\right|, \quad \hat{N}_{i, j}=\left|\overline{\mathscr{N}}_{j, i}\right|,
$$

and also

$$
N=2 N_{i, j}+1, \quad \bar{N}=\tilde{N}_{i, j}+\hat{N}_{i, j},
$$

where the indices are avoided in the latter definitions to improve the readability. 
The dynamics of the $i j$ system, perturbed by the unknown agents, is

$$
\begin{aligned}
e_{i, j}^{+}= & \left(A+B K_{i, j}+B K_{j, i}\right) e_{i, j}+\sum_{k \in \mathscr{N}_{i, j}}\left(B K_{i, k} e_{i, k}-K_{j, k} e_{j, k}\right)+ \\
& +\sum_{k \in \overline{\mathscr{N}}_{i, j}}\left(B K_{i, k} e_{i, k}\right)-\sum_{k \in \overline{\mathscr{N}}_{j, i}}\left(B K_{j, k} e_{j, k}\right),
\end{aligned}
$$

where the terms of the first summation are known, being related to the neighbours of $j$-th agent known be the $i$-th and vice-versa, while only a bound on the values of those of the other summations is known. In fact, since they are neighbours of the $j$-th and the $i$-th agents, they are bounded by the radius $r$, that is

$$
\begin{array}{ll}
\left\|T e_{i, k}\right\|_{2} \leq r, & \text { if } k \in \overline{\mathscr{N}}_{i, j}, \\
\left\|T e_{j, k}\right\|_{2} \leq r, & \text { if } k \in \overline{\mathscr{N}}_{j, i} .
\end{array}
$$

The problem addressed in this paper can be stated as below.

Problem 1 Design a procedure to find at each step the control gains $K_{n, m}$ in (7) such that the the following algebraic relation is satisfied

$$
\left\|T e_{i, j}^{+}\right\|_{2}<r,
$$

for all $(i, j) \in \mathscr{N}$ i.e. $\left\|T e_{i, j}\right\|_{2}<r$ and for all $e_{i, k}$ and $e_{j, k}$ such that (8) holds.

\section{Set theory based condition for topology preservation}

For notational convenience, we employ the following definitions:

$$
\begin{aligned}
& \check{K}_{i, j}=K_{i, j}+K_{j, i}, \\
& \tilde{K}_{i, k}=K_{i, k}, \quad \hat{K}_{j, k}=-K_{j, k}, \quad \text { if } k \in \mathscr{N}_{i, j}, \\
& \tilde{G}_{i, k}=K_{i, k}, \quad \text { if } k \in \mathscr{\mathscr { N }}_{i, j}, \\
& \hat{G}_{j, k}=-K_{j, k}, \quad \text { if } k \in \mathscr{\mathscr { N }}_{j, i} \text {. }
\end{aligned}
$$

Definition 2 Denote with $\mathbf{E}_{i, j} \in \mathbb{R}^{n N}$ the vector obtained concatenating $e_{i, j}$ with all $e_{i, k}$ and $e_{j, k}$ where $k \in \mathscr{N}_{i, j}$ and $\bar{E}_{i, j} \in \mathbb{R}^{n \bar{N}}$ the vector obtained concatenating all $e_{i, k}$ where $k \in \overline{\mathscr{N}}_{i, j}$ and $e_{j, k}$ where $k \in \overline{\mathscr{N}}_{j, i}$. Define

$$
\begin{aligned}
\Delta_{i, j} & =\left[A+B \check{K}_{i, j}, B \tilde{K}_{i, 1}, \ldots, B \hat{K}_{j, N}\right], \\
\Gamma_{i, j} & =\left[B \tilde{G}_{i, 1}, \ldots, B \hat{G}_{j, \hat{N}}\right] .
\end{aligned}
$$

A change of variable is introduced before proceeding. Define

$$
\begin{aligned}
& Z=\mathscr{D}([T, \ldots, T]) \mathbf{E}_{i, j} \in \mathbb{R}^{d N}, \\
& \bar{Z}=\mathscr{D}([T, \ldots, T]) \bar{E}_{i, j} \in \mathbb{R}^{d \bar{N}}, \\
& \Delta=T \Delta_{i, j} \mathscr{D}([T, \ldots, T])^{\dagger} \in \mathbb{R}^{d \times d N}, \\
& \Gamma=T \Gamma_{i, j} \mathscr{D}([T, \ldots, T])^{\dagger} \in \mathbb{R}^{d \times d \bar{N}},
\end{aligned}
$$


and notice that the (right) pseudo inverse matrices exist from the Definition 1. Define also the matrix

$$
D_{k}=\mathscr{D}\left(\left[0_{d}, \ldots, I_{d}, \ldots, 0_{d}\right]\right)
$$

with $k \in \bar{N}$. Using the Definition 2, the quantity $T e_{i, j}^{+}$is given in terms of the matrices $\Delta$ and $\Gamma$ as

$$
T e_{i, j}^{+}=T \Delta_{i, j} \mathbf{E}_{i, j}+T \Gamma_{i, j} \bar{E}_{i, j}=\Delta Z+\Gamma \bar{Z},
$$

where $\mathbf{E}_{i, j}$ is the vector of known distances and the uncertainties are given by the term $\bar{E}_{i, j}$ which is such that

$$
\bar{E}_{i, j}^{\top} \mathscr{D}([T, \ldots, T])^{\top} D_{k} \mathscr{D}([T, \ldots, T]) \bar{E}_{i, j} \leq r^{2}
$$

for all $k \in \bar{N}$, or, equivalently

$$
\bar{Z}^{\top} D_{k} \bar{Z} \leq r^{2}, \quad \forall k \in \bar{N},
$$

from (8). Then, from (11) and (12), the algebric constraint (9) is equivalent to

$$
[\Delta Z+\Gamma \bar{Z}]^{\top}[\Delta Z+\Gamma \bar{Z}]<r^{2}
$$

from (12).

A first constraint on the matrix $\Gamma$ can be posed in order to ensure that there is not an admissible realization of the uncertainty $\bar{Z}$ which would lead to the certain break of the link between the $i$-th and the $j$-th agent.

Assumption 1 The matrix $\Gamma$ is such that $\operatorname{Tr}\left(\Gamma^{\top} \Gamma\right)<d$.

Assumption 1 provides a necessary condition for the Problem 1 to have a solution. In fact, the violation of Assumption 1 implies the existence of an admissible $\bar{Z} \in \mathbb{R}^{d \bar{N}}$, i.e. satifying (13), such that (14) is not feasible, as proved below.

Proposition 1 (Necessary condition) If Assumption 1 does not hold, then there is $\bar{Z} \in$ $\mathbb{R}^{d \bar{N}}$ such that (13) holds and

$$
[\Delta Z+\Gamma \bar{Z}]^{\top}[\Delta Z+\Gamma \bar{Z}] \geq r^{2}
$$

for all $Z \in \mathbb{R}^{d N}$ and $\Delta$.

A computation oriented condition ensuring the satisfaction of Assumption 1 is presented in the following remark. Such relaxed condition is employed in the next section to provide sufficient conditions for Problem 1 to have solutions.

Remark 1 The matrix $\Gamma$ satisfies Assumption 1 if there exists $\Lambda=\mathscr{D}\left(\left[\lambda_{1} I_{d}, \ldots, \lambda_{\bar{N}} I_{d}\right]\right)$ with $\lambda_{k} \geq 0$ and $\sum_{k \in \mathbb{N}_{\bar{N}}} \lambda_{k} \leq 1$ such that

$$
\Gamma^{\top} \Gamma<\Lambda
$$


The fact that the condition presented in Remark 1 implies the satisfaction of Assumption 1 follows directly from the fact that (16) implies $\operatorname{Tr}\left(\Gamma^{\top} \Gamma\right)<\operatorname{Tr}(\Lambda)$.

Hence, no loss of generality is due to Assumption 1 since in case it is not satisfied, there is no guarantee that the successor $e_{i, j}^{+}$would satisfy the constraint (9). The main contribution, namely the sufficient condition for the constraint (9) to hold, is stated in the following theorem.

Theorem 1 Condition (14) is satisfied for every $\bar{Z} \in \mathbb{R}^{d \bar{N}}$ fulfilling (13), if there exist $\Lambda=\mathscr{D}\left(\left[\lambda_{1} I_{d}, \ldots, \lambda_{\bar{N}} I_{d}\right]\right)$ with $\lambda_{k} \geq 0$, for all $k \in \mathbb{N}_{\bar{N}}$, such that

$$
\left[\begin{array}{ccc}
r^{2}-r^{2} \sum_{k=1}^{\bar{N}} \lambda_{k} & 0 & Z^{\top} \Delta^{\top} \\
0 & \Lambda & \Gamma^{\top} \\
\Delta Z & \Gamma & I_{d}
\end{array}\right]>0 .
$$

Remark 2 Geometrically, the value $\sum_{k=1}^{\bar{N}} \lambda_{k}$ represents a measure of the ellipsoid (determined by $T^{\top} T$ ) bounding the generated uncertainty. If such a value is greater or equal than 1, then the uncertainty is bounded by an ellipsoid of radius greater or equal than $r$ and the constraint (14) could be violated. In fact, the existence of an admissibile $\Lambda$ satifying (??) and (??) implies the satisfation Assumption 1, condition necessary for the Problem 1 to admit a solution.

\section{Illustrative example}

We consider a distributed system composed by three agents moving on a two dimensional space with same dynamics. Every agent is supposed to be able to select the variation of its velocity. We suppose that the 2-nd agent is a common neighbour of both the 1-st and the 3-rd one, which are not neighbours each other. Then modelling the input as a velocity variation, or equivalently, the variations along the two Cartesian coordinates, the dynamics of the $i$-th agent along the $x$ axis is given by

$$
\left\{\begin{array}{c}
p_{i}^{x}(k+1)=p_{i}^{x}(k)+t v_{i}^{x}(k), \\
v_{i}^{x}(k+1)=v_{i}^{x}(k)+u_{i}^{x}(k),
\end{array}\right.
$$

for every $i \in \mathbb{N}_{3}$, where $p_{i}^{x}$ is the position, $v_{i}^{x}$ the velocity, $u_{i}^{x}$ the control input and $t$ the sampling time. The dynamics along the $y$ axis are clearly analogous. Consider the system (18) and notice that the effect of the input at time $k$ has no influence on the state $p_{i}^{x}$ at time $k+1$. In fact, any algebraic condition involving the position $p_{i}^{x}$ of the systems at $k+1$ would not depend on the control action $u_{i}^{x}$ at time $k$. From the computational point of view, every constraint concerning only the agents positions, would lead to LMI conditions independent on the variable $K_{i, j}$. Then the results provided in Theorem 1 are not applicable directly in this case. On the other hand, the control $u_{i}^{x}(k)$ affects the position (and the velocity) at time $k+2$. The topology preservation constraint can be imposed on the states at $k+2$, knowing that nothing can be done in order to prevent its violation at time $k$. The dynamics of $p_{i}^{x}$ at $k+2$ is given by

$$
p_{i}^{x}(k+2)=p_{i}^{x}(k+1)+t v_{i}^{x}(k+1)=p_{i}^{x}(k+1)+t v_{i}^{x}(k)+t u_{i}^{x}(k),
$$


and then the overall dynamics of the motion of the $i$-th agent along the $x$ axis is given by the linear system (1) with matrices

$$
\hat{A}=\left[\begin{array}{lll}
1 & 0 & t \\
0 & 1 & t \\
0 & 0 & 1
\end{array}\right], \quad \bar{B}=\left[\begin{array}{l}
t \\
0 \\
1
\end{array}\right]
$$

where the sampling time $t$ has been chosen equal to 0.05 . Then the whole dynamics of every agent is

$$
A=\left[\begin{array}{cc}
\bar{A} & 0 \\
0 & \bar{A}
\end{array}\right], \quad B=\left[\begin{array}{cc}
\bar{B} & 0 \\
0 & \bar{B}
\end{array}\right]
$$

where $\left[p_{i}^{x}(k+1), p_{i}^{x}(k), v_{i}^{x}(k), p_{i}^{y}(k+1), p_{i}^{y}(k), v_{i}^{y}(k)\right]^{T}$ is the state.

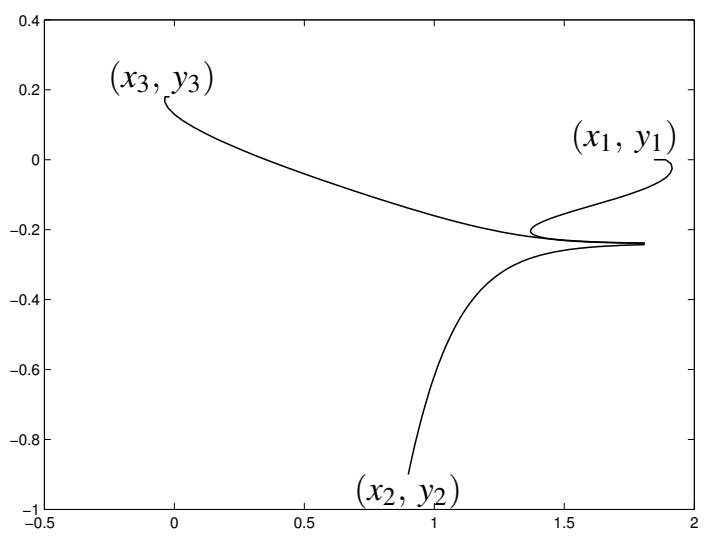

Figure 1: Trajectories of the three agents in the plane.

We pose now a constraint on the distance between the first and the second agents in $k$ and $k+1$, that is condition (9) with

$$
\bar{T}=\left[\begin{array}{lll}
1 & 0 & 0 \\
0 & 1 & 0
\end{array}\right], \quad T=\left[\begin{array}{cc}
\bar{T} & 0 \\
0 & \bar{T}
\end{array}\right],
$$

and $r=4$. The same condition is imposed to maintain the neighbourhood between the second and the third agents. Finally, notice that since the value of the $p_{i}^{x}(k+1)$ is unknown at time $k$, then the feedback should concern only the velocities and the position at time $k$. We solve an optimization problem with feedback gains $K_{i, j}=\alpha_{i, j} K$ where

$$
K=\left[\begin{array}{llllll}
0 & 1 & 1 & 0 & 0 & 0 \\
0 & 0 & 0 & 0 & 1 & 1
\end{array}\right]
$$

for $(i, j)=(1,2)$ and $(i, j)=(2,3)$. The optimization objective has been to compute the feedback gains satisfying the condition presented in Theorem 1 minimizing the euclidean measure of the error. A set of trajectories in the plane are shown in Figure 1 , obtained for initial conditions $x_{1}(0)=1.8, v_{1}^{x}(0)=0.9, x_{2}(0)=0.9, y_{2}(0)=-0.9$, 
$v_{3}^{x}(0)=-0.36, y_{3}(0)=0.18$ and 0 for all the other states. Notice that, as the control objective is the minimization of the norm of the errors, the systems states mismatches converge to zero. Moreover, the algebraic constraint is satisfied along the whole trajectories, also at the first instants, which are the most critical ones as the system initial conditions are close to the boundary of the feasible region, see Figure 2.

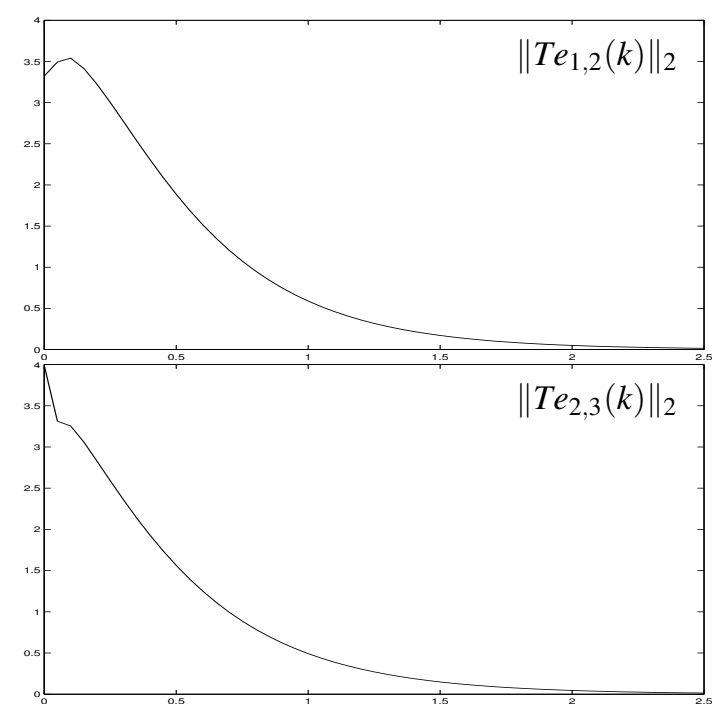

Figure 2: Time evolutions of $\left\|T e_{1,2}(k)\right\|_{2}$ and $\left\|T e_{2,3}(k)\right\|_{2}$.

\section{Conclusion and further works}

The paper proposes a set theory based design for the interconnection topology preservation in multi-agent systems. The method allows one to preserve the connectivity of the network which is an essential ingredient for the decentralized control. A necessary and a sufficient condition for the feedback control to ensure the topology preservation are given in form of convex LMI. Many direction of possible further developments can be based on the results presented in the paper. Convergence and contractiveness properties, basin of attraction estimation, robust control design, model predictive control, for instance, are possible line of research, besides the application to real systems in problems of consensus and flocking.

\section{References}

[1] T.C. Aysal, M.E. Yildriz, A.D. Sarwate, and A. Scaglione. Broadcast gossip algorithms for consensus. IEEE Transactions on Signal Processing, 57:27482761, 2009. 
[2] G. Basile and G. Marro. Controlled and conditioned invariant subspaces in linear system theory. Journal of Optimization Theory and Applications, 3:306-315, 1969.

[3] G. Basile and G. Marro. On the robust controlled invariant. Systems and Control Letters, 9:191-195, 1987.

[4] G. Basile and G Marro. Controlled and Conditioned Invariants in Linear system Theory. Prentice Hall, 1992.

[5] A. Ben-Tal and A. Nemirovski. Lectures on modern convex optimization. MPSSIAM, 2001.

[6] D. P. Bertsekas. Infinite-time reachability of state-space regions by using feedback control. IEEE Trans. on Automatic Control, 17:604-613, 1972.

[7] D. P. Bertsekas and I. B. Rhodes. On the minimax reachability of target sets and target tubes. Automatica, 7:233-247, 1971.

[8] D. P. Bertsekas and I. B. Rhodes. Recursive state estimation for a set-membership description of uncertainty. IEEE Trans. on Automatic Control, 16:117-128, 1971.

[9] F. Blanchini. Ultimate boundedness control for discrete-time uncertain systems via set-induced Lyapunov functions. IEEE Trans. on Automatic Control, 39:428433, 1994.

[10] F. Blanchini. Set invariance in control. Automatica, 35:1747-1767, 1999.

[11] F. Blanchini and S. Miani. Set-Theoretic Methods in Control. Birkhäuser, 2008.

[12] V. D. Blondel, J. M. Hendrickx, and J.N. Tsitsiklis. On krause's multi-agent consensus model with state-dependent connectivity. IEEE Transactions on Automatic Control, 54(11):2586-2597, 2009.

[13] V.D. Blondel, J.-L. Guillaume, R. Lambiotte, and E. Lefebvre. Fast unfolding of communities in large networks. Journal of Statistical Mechanics: Theory and Experiment, , P10008(10), 2008.

[14] S. Boyd, L. El Ghaoui, E. Feron, and V. Balakrishnan. Linear Matrix Inequalities in system and control theory. SIAM, 1994.

[15] S. Boyd, A. Ghosh, B. Prabhakar, and D. Shah. Randomized gossip algorithms. IEEE Transactionson Information Theory, 52(6):2508-2530, 2006.

[16] S. Boyd and L. Vandenberghe. Convex Optimization. Cambridge University Press, 2004.

[17] R. Carli, F. Fagnani, A. Speranzon, and S. Zampieri. Communication constraints in the average consensus problem. Automatica, 44(3):671-684, 2008.

[18] F. L. Chernousko. Optimal ellipsoidal estimation of dynamic systems subject to uncertain disturbances. Cybernetics and systems analysis, 38:221-229, 2002. 
[19] J. D. Glover and F. C. Schweppe. Control of linear dynamic systems with set constrained disturbances. IEEE Transactions on Automatic Control, 16(5):411423, 1971.

[20] R. Hegselmann and U. Krause. Opinion dynamics and bounded confidence models, analysis, and simulation. Journal of Artificial Societies and Social Simulation, 5(3), 2002.

[21] A. Jadbabaie, J. Lin, and A.S. Morse. Coordination of groups of mobile autonomous agents using nearest neighbor rules. IEEE Transactions on Automatic Control, 48:988-1001, 2003.

[22] I. Kolmanovsky and E. G. Gilbert. Theory and computation of disturbance invariant sets for discrete-time linear systems. Mathematical Problems in Engineering, 4:317-367, 1998.

[23] D. Maksarov and J. P. Norton. State bounding with minimal volume ellipsoids. In Proceedings of UKACC International Conference on Control '96, 1996.

[24] S. Martin and A. Girard. Sufficent conditions for flocking via graph robustness analysis. In In Proc. of IEEE Conference on Decision and Control, 2010.

[25] D.Q. Mayne, J.B. Rawlings, C.V. Rao, and P.O.M. Scokaert. Constrained model predictive control: Stability and optimality. Automatica, 36:789-814, 2000.

[26] L. Moreau. Stability of multiagent systems with time-dependent communication links. IEEE Transactions on Automatic Control, 50:169-182, 2005.

[27] I.-C. Morărescu and A. Girard. Opinion dynamics with decaying confidence: application to community detection in graphs. IEEE Transactions on Automatic Control, 56(8):1862-1873, 2011.

[28] K. Nawrotzki. Distributed subgradient methods for multi-agent optimization. IEEE Transactions on Automatic Control, 54(1):48-61, 2009.

[29] R. Olfati-Saber. Flocking for multi-agent dynamic systems: Algorithms and theory. IEEE Transactions on Automatic Control, 51:401-420, 2006.

[30] R. Olfati-Saber and R.M. Murray. Consensus problems in networks of agents with switching topology and time-delays. IEEE Transactions on Automatic Control, 49:1520-1533, 2004.

[31] W. Ren and R.W. Beard. Consensus seeking in multiagent systems under dynamically changing interaction topologies. IEEE Transactions on Automatic Control, pages 655-661, 2005.

[32] R. T. Rockafellar. Convex Analysis. Princeton University Press, USA, 1970.

[33] R. Schneider. Convex bodies: The Brunn-Minkowski theory, volume 44. Cambridge University Press, Cambridge, England, 1993. of Encyclopedia of Mathematics and its Applications. 
[34] F. C. Schweppe. Recursive state estimation: Unknown but bounded errors and system inputs. IEEE Trans. on Automatic Control, 13:22-28, 1968.

[35] J.N. Tsitsiklis. Problems in Decentralized Decision Making and Computation. $\mathrm{PhD}$ thesis, PhD Thesis,Massachusetts Institute of Technology, 1984.

[36] J.N. Tsitsiklis, D.P. Bertsekas, and M. Athans. Distributed asynchronous deterministic and stochastic gradient optimization algorithms. IEEE Transactions on Automatic Control, 31:803-812, 1986.

[37] H. S. Witsenhausen. A minimax control problem for sampled linear systems. IEEE Trans. on Automatic Control, 1:5-21, 1968.

[38] H. S. Witsenhausen. Sets of possible states of linear systems given perturbed observations. IEEE Trans. on Automatic Control, 13:556-558, 1968. 